\title{
Power-Awareness Extensions for Network Testbeds
}

\author{
Joseph Chabarek \\ University of Wisconsin-Madison \\ Email: jpchaba@cs.wisc.edu
}

\author{
Paul Barford \\ University of Wisconsin-Madison \\ Qualys, Inc. \\ Email: pb@cs.wisc.edu
}

\begin{abstract}
The escalation in power consumption of networking and communications equipment is of concern to technologists and environmentalists alike. Understanding how and when networking devices consume power is complicated by their lack of instrumentation. Furthermore, standard networking devices are not typically flexible enough to support experiments with new techniques for reducing power consumption. In this paper, we describe a set of extensions for Linux-based commodity switches that enable a wide range of power-aware experiments in laboratory testbeds. The extensions are based on the requirements for high fidelity in power measurement and in modulation of key subsystems. Our implementation includes two key capabilities: a flexible power consumption model and a traffic shaper that enable emulation of a wide range of power-aware hardware and protocols. To validate our power-awareness extension and demonstrate their capabilities and utility, we built a testbed composed of simple, Linux-based switches. First, we show that the most simple configuration of our emulation extensions report power consumption consistent with what can be measured with an external power meter. Next, we conduct a series of experiments on power consumption when bandwidth is scaled directly to performance demands. Our results confirm our hypothesis that a finer grained approach yields more power savings when the transition cost is low and traffic varies.
\end{abstract}

\section{INTRODUCTION}

Networking and communication devices such as Ethernet switches and IP routers are found in office buildings, points of presence, colocation and data centers throughout the world. The ubiquity and importance of these devices coupled with their increasing power density [9] and associated cooling costs are strong motivations for designing future systems and protocols that are power-aware and power efficient.

The predominant approach followed by the published research papers on power-awareness in networking and communications devices is to investigate new methods for turning off systems entirely or for turning off major subsystems such as line cards (e.g., [6], [9]-[11]). While this general approach makes a good deal of sense especially in contrast to the "always on at maximum speed" configuration, which is typical in today's equipment, it only represents a small portion in a much larger design space for introducing power-awareness in networking and communications systems.

There are several challenges in experimental study of powerawareness in networking and communications equipment. First, there are relatively few ways in which one can exert finegrained control in the current generation of communications devices such that they can be made to behave in a power conserving fashion. For example, if one assumes that power consumption by a switch or router is directly correlated with the amount of traffic it is forwarding and that bandwidth could be modulated in a fine-grained fashion, then one could experiment with traffic prediction algorithms and their associated impact on power consumption. However, to the best of our knowledge no commercial communications devices allow bandwidth and power to be controlled in this fine-grained fashion. Second, most communications devices have a very limited capability to report the power they consume. Typically, only an overall power consumption SNMP MIB variable is available on a device (e.g., the cdpCachePowerConsumption MIB reports total power consumed in milliwatts on some Cisco devices). Unfortunately, coarse-grained measures like these do not allow for the details of power consumption in subsystems to be assessed, which is critical to the development of enhanced power-awareness. Third, external monitoring via power meters is only sufficient to capture gross level effects $e . g$. turning off subsystems such as line cards. This is because devices are built with power systems that smooth and buffer power draw from the socket.

In this paper, we address the problem of how to conduct a broad range of testbed-based experiments on power-awareness in networking and communications devices. Our key observations are 1) that there is an inherent tradeoff between performance and saving power (i.e., by turning components off or operating at reduced levels), and 2) that fine-grained measurement of the effects of modulation of subsystems is essential. Our objective is to develop a set of capabilities for devices that are found in openly available network testbeds (e.g., [4] and experimental network systems (e.g., [13]) that enable the analysis of the tradeoffs between performance and power modulation with fine-grained measurement in a flexible and realistic fashion.

Our specific contribution is the implementation of a set of extensions for Linux-based Ethernet switches that enable power-awareness experiments. The first component of our implementation is a monitor that reports a highly accurate model based estimation of fine-grained power usage of a target device in real time. The key element of our monitor is that it can report the total system power usage and it also includes a power consumption model, which can be parameterized to report the aggregate power savings that could be realized if a specific subsystem or protocol had an envisioned (but not yet implemented) power awareness capability. The model enables power metering to be tied directly to a device's dynamic behavior and therefore falls in a rather unique space between pure simulation and device-based emulation. 
The second component of our implementation is a traffic shaper that enables the characteristics of traffic flowing through a target switch to be changed to emulate the effects of an envisioned power-aware protocol or capability. As noted above, our assumption is that power-awareness and efficiency, in many cases, will come at the potential cost of performance. The traffic shaper enables packet rates to be throttled in different ways using a variety of queue types, and can be tied directly to our power consumption monitor.

We validate and demonstrate our implementation through a series of laboratory-based experiments. We selected a simple target platform on which to build our testbed and load our extensions - the Linksys WRT54GL Wireless Router [1]. The first validation experiment measured the power consumption of a single switch using a power cord clamp versus what was reported by our emulation power monitor configured to report chassis power consumption (i.e., no traffic through the switch). The results of this simple experiment show that our monitor captures the average power consumption accurately. In the second validation experiment, we configured our emulation traffic shaper to limit bandwidth to a fixed rate. Using the IPERF traffic generator, we show that indeed the shaper is responsive and correct. Next, we conducted a set of experiments with adapting bandwidth and power consumption to traffic load versus an "always on at maximum speed" configuration. Our intention in these tests was to demonstrate the utility and capabilities of our extension, not to make a case for quantitative results. Using variable packet traffic, the results show the power savings that could be achieved by modulating bandwidth.

\section{RELATED WORK}

Prior studies that are most closely related to our own fall into two categories: those that describe network simulation or emulation capabilities and those that inform the design of our extensions. While we are not aware of any other experimental environment that can specifically support network power-awareness experiments, there has been a great deal of prior work on developing general tools and environments for experimental network research. Widely used examples include the ns2 simulator [5], the Emulab testbed [4] and the Planetlab testbed [7]. The wireless and mobility extensions developed for ns2 are an example of enhancing a test environment to support a new class of experiments [8], which is the general goal of our work. Another related experimental system is OpenFlow [13], which enables experimental protocols to be run on commodity devices. Our extensions were designed to complement OpenFlow's capabilities with power monitoring and fine-grained traffic shaping.

A number of studies inform the design of our extensions by identifying sleeping and rate adaptation as two modalities for energy savings [9], [10], [14]. These studies often use simulation-based analysis to assess the impact of network power-awareness techniques. While the results of these studies highlight the potential for power-awareness, [14] specifically acknowledges the lack of ability to conduct experiments with actual hardware. New classes of commercial systems may offer some flexibility in this regard [15], although they are not readily available in testbeds at this time. Finally, Heller et al. use a model based approach to assess data center routing methods with OpenFlow in [11]. Our extensions implement the device model on the testbed switch in real time allowing for interaction between device modulation to save power and live network traffic.

\section{AN EXPERIMENTAL FRAMEWORK FOR POWER-AWARE SWITCHING}

The objective of our work is to enhance Linux-based switches with the ability to support diverse experiments on new power-aware networking protocols and configurations. With this capability, we envision that existing network testbeds could be used for power-awareness experiments and that new, low cost power-awareness testbeds could be build from simple devices such as the Linksys device described in Section IV-A. It is important to note that there are a growing number of Linux-based switches, which makes our enhancements broadly applicable.

\section{A. Requirements for Testbed Switches that Support Power- Aware Experiments}

We are motivated by the notion of power proportionality [14], but also want to support a broad notion of how and when power might be consumed on a network device. To that end, we specify the following general requirements for switches are used in power-aware experiments:

1) Support for fine-grained modulation of traffic streams.

2) Support for flexible assignment of power consumption.

3) Support for fine-grained measurement of power consumption.

Support for fine-grained modulation of traffic streams recognizes that power proportionality may call for packet transmission at different rates or at different levels of service in contrast to "always on at maximum rate". Support for flexible assignment of power consumption recognizes that the effects of new power proportional methods must be reflected in detailed reporting of the power consumed. Support for finegrained measurement recognizes that detailed power consumption reports from experiments must be gathered for subsequent analysis.

Unfortunately, the current generation of Linux-based switches does not satisfy any of these requirements. We are aware of no switches that allow flexible power proportional use of subsystems or expose fine-grained power consumption (although worst case power consumption is typically available from datasheets). To fill this gap and facilitate the evaluation of the the tradeoff between power savings and performance we have extended a commodity switch with an on-device real time power model that is parameterized with measurements and enabled flexible simulation of subcomponent modulation of the device with traffic shaping. 


\section{B. Power-Aware Extensions}

With our requirements and the limitations of current systems in mind, we developed a set of power-awareness extensions for Linux-based switches. At the core of the extensions is a flexible model for reporting power consumption that has two distinct components. The first component of the model enables specification of the baseline power consumption for the major subsystems of a switch such as its chassis, cooling fans, ports, control fabric, etc. For example, one might envision a switch with a new lower level baseline for power consumption for the chassis and cooling fan. Power consumption reporting for the baseline configuration of the model depends on the active set of subsystems during an experiment (it can also simply be set as a constant value). The second component of the model enables specification of the power consumption based on activity in different components of the switch such as the $\mathrm{CPU}$, busses, the switching fabric, ports, etc. For example, one might envision a switch with a power proportional switching fabric, thus experiments must reflect the power consumption based on the actual use of the fabric. Power consumption reporting for the activity on the switch will be proportional to traffic observed in /proc/net/dev during an experiment.

We argue that this model enables a broad range of powerawareness experiments. Beyond the obvious examples of turning subsystems off or power-proportionality, there are more subtle effects that can be examined. For example, current switches have a long latency in component modulation and turning them on can incur a "spike" in power consumption. Thus, experiments with modulation of current switches must be able to consider the latency in powering down/up a component, the possibility of packet loss during a burst of traffic and the brief increase in power consumption to initialize. Our power model can accommodate these effects, but must be coupled with the second component of our extensions a traffic shaper, in order to address the corresponding impact on traffic. Our framework includes a simple configuration language to parameterize the model.

Closely coupled to our power consumption model is a flexible monitor that enables power measurements to be gathered in a fine-grained fashion. The monitor is configured to gather estimated power consumption from the device model at a specified frequency and to record the measurements in a local $\log$ or report to remote data collectors. For remote reporting, the device runs a SNMP agent defined by net-snmp [2]. The power monitor can write variables to the SNMP MIB using an AgentX module. The granularity of real-time reporting is influenced by the duration between reports set by the timers in the monitor, activities in the traffic shaping filter, and the CPU capability of the device.

The second component of our power-aware extensions is a flexible traffic shaper that modulates the profile of packet emissions. The traffic shaper is implemented to have the effect of reducing bandwidth, prioritizing packet emissions or to enforce hard ceilings on packet emissions that are representative of an overloaded device dropping packets. Our implementation is based on the traffic control (TC) utility in Linux. TC enables a hierarchical token bucket (HTB) to be established as "queue disk" on an egress queue or an intermediary queuing device if ingress filtering is required. The HTB uses a hierarchically organized set of token bucket filters to slow traffic over an interface to a desired rate. This algorithm uses a token abstraction to time the service of a queue and potentially allows short bursts over the ceiling rate, but is efficient and tunable. Each HTB can be configured to establish sub-classes that specify the transmission of packets at a specified rate and to establish a burst rate and a rate ceiling. This implementation enables a switch to manage the details of traffic characteristics in a fine-grained fashion in real time. We developed our implementation to be highly efficient and thereby have a minimal impact on the performance of the target system. We establish the hierarchy of buffers a priori and then change the filters classifying traffic as belonging to a particular class during an experiment.

\section{Implementation of Power-Aware Extensions}

In addition to the power model, monitor, and traffic shaper we include an experiment scenario script for configuration of each of the components. A scenario is a policy that changes component settings in response to an input such as the traffic load on the switch, or an external prompt. For example, a scenario that implements bandwidth scaling - changing the speed of a link according to how much traffic has transited the link over a given time - will periodically measure the traffic through a given interface and change the running configuration to a lower link speed if possible (e.g., interface 1 at $100 \mathrm{Mbps}$ to interface 1 at $10 \mathrm{Mbps}$ ).

Our implementation ties together the Linux TC utility for traffic shaping with a custom power model, monitor, and scenarios. Our extensions are written in roughly 1000 lines of $\mathrm{C}++$. The code used in the experiments in the following sections is compiled for a MIPS target. The extensions will run on a wide variety of Linux kernels since all of the functions they use have been standard in distributions for some time.

The framework has been designed to have a minimal performance footprint. For example, the power monitor uses sleeping instead of polling, and the time between reports can be set to a larger duration if the target device is under high load.

\section{Building A Power-Awareness Testbed}

To validate and demonstrate the capabilities of our powerawareness extensions, we built a small experimental testbed with low-cost home routers. In the following sections, we describe the target platform used in the testbed, it's configuration and our validation experiments.

\section{A. Target Device Platform}

We selected the Linksys WRT54GL switch [1] as the target platform for implementation and evaluation of our extensions. The reason for this selection is that these Linux-enabled devices are inexpensive, relatively capable and easy to use. The 
switch has a $200 \mathrm{MHz}$ CPU, 16MB RAM and 4MB of flash memory. It has 5 wired ports plus 1 wireless port (we disabled the wireless port for our tests) and uses a shared bus to forward packets between ports, and supports microsecond timers. In simple IPERF tests we found that the bus can forward a maximum of roughly 200Mbps, which is confirmed on the device datasheet. We use the Kamikaze Linux distribution provided by the OpenWRT [3] project to deploy an operating system that is amenable to development. The combination of system capabilities, programmability and port density make the switch an attractive experimental platform and enable highly functional power-awareness testbeds to be constructed at modest cost.

There are several limitations with the system. In order to gather packet statistics from the Linux kernel and shape traffic we had to configure the switch to give each of 5 ports a unique VLAN and then we bridge traffic between VLANs (the device supports up to 15 VLAN tags). With our packet shaping enabled, the maximum line rate is roughly $50 \mathrm{Mbps}$ due to the CPU bottleneck caused by the additional load from maintaining the HTB. While this is a substantial overhead the purpose of our testbed is to explore the tradeoffs in poweraware switching rather than achieve the highest possible line rate. Additionally, we are bound by the CPU for the maximum frequency that we can update the model and report from the monitor causing drift in model updates and reports from the monitor when the system is under high load. To overcome this challenge, we timestamp each report from the monitor to give the experimenter an idea of the time period being reported.

\section{B. Power Model}

For the validation experiments reported below, we begin with a simple power model configuration that can be verified by measurements of the WRT54GL target device using an external power meter. The model is implemented as a daemon on the test device. In our model configuration, we assume that the total power consumption of the device is the sum of the consumption of the components. We begin by adding two components to the model, the ports and the chassis as shown in Table I. This model specifies a fixed cost for the idle state of each component. We also specify a fixed cost for the "full load" state of ports (i.e., one in which there is no proportionality of power consumption with load). Our rational for this configuration is due to observations in prior work that the power profile of contemporary switches is not very sensitive to the amount of traffic they are forwarding [9], [12].

Selection of the fixed values for the power model are based on meter-based measurements of the WRT54GL. We use a Fluke 189 power meter with a power cord voltage clamp, we assume a power factor of 1 . The measurements should be seen as a relative comparison rather than a qualitative result. We begin by measuring the power consumption when the device is powered but all links are down. We call this the "chassis" cost which includes the CPU, bus, and memory. We measure power consumption by bringing up a port and observing the difference in power consumption versus the chassis cost. Finally, we found that when traffic is added at maximum rate, there was a modest increase in total power consumption as reflected in Table I.

TABLE I

MEASUREMENT BASED MODEL PARAMETERS

\begin{tabular}{|l|l|}
\hline Component & Power Usage \\
\hline Chassis & Fixed @ 2.88W \\
Port@ $100 \mathrm{Mbps}+$ no load & Fixed @ $0.24 \mathrm{~W}$ \\
Chassis +3 ports + full load & Fixed @ 4.2W \\
\hline
\end{tabular}

This is one model that represents the WRTG54GL switch type. Additional switches can be tested with a similar measurement based parameterization or use a device model augmented with current or future power estimates based on a researcher's knowledge of the hardware components.

\section{Validation Experiments}

The goal of our validation experiments is to demonstrate the basic functions of our extensions running on the Linksys switch with the aforementioned simple configuration. The basic functions include reporting and measuring power consumption through the model/monitor respectively, and traffic shaping.

The first experiment is very simple, but an important benchmark for the basic functionality of our system. The goal is to use the simple model and monitor to gather power measurements for the chassis and chassis + ports in a static configuration. We expect the values reported by the model and gathered via the power meter to be consistent. Examination of the time series of power measurements from the model shows a stable value while there is fluctuation in power consumption reported by the meter. When the device is not switching packets, the maximum difference observed between the model and the measurement averaged power consumption over $100 \mathrm{~ms}$ bins is less than $1 \%$. As load increases so does the variability of the measurements compared to the model due to the model being overly simple. We could achieve a tighter correlation by using the monitor to track dynamic activity such as CPU, memory, etc. and then enhancing the model to reflect dynamic consumption by subsystems. In future work we plan to refine the power model by adding components validated from measurements or component specifications.

Next, we validate the capabilities of the traffic shaping component of our system. This experiment is designed to show how rate limiting can be imposed on a traffic stream through the use of our HTB-based queues. The experiment uses IPERF configured to send packets through the switch test running at $10 \mathrm{Mbps}$. After 2 seconds of sampling, the scenario enables the traffic shaping utility to set a ceiling rate of $5 \mathrm{Mbps}$ on the buffer thereby scaling down the link speed. We gather packet rate data in this experiment using snapshots of the adapter usage as reported in /proc/net/dev. Figure 1 shows traffic rates extracted from data collected in bins over a tenth of a second. We see exactly what is expected: the transmit rate begins at roughly $10 \mathrm{Mbps}$ and then goes down to $5 \mathrm{Mbps}$ when the 


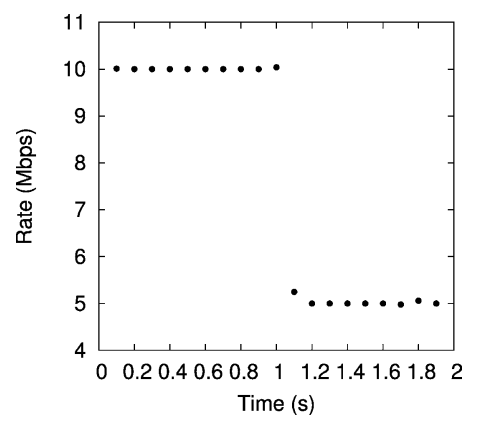

Fig. 1. Traffic shaping results using the $t c$ tool. Transmit statistics reported from the /proc/net/dev API every tenth of a second.

traffic shaper is enabled at the 1 second mark. Much more complex traffic shaping is possible with our implementation and in future work, we plan to develop a number of standard configurations for our system.

\section{AdAptive BAndwidth Scaling}

The next set of experiments demonstrate the ability of our system to support investigations on the power proportional tradeoffs in adapting the line rate of a port to the amount of transmitted traffic. We consider three different model configurations for these tests, in each configuration the chassis and two ports are active. The first configuration sets each port to the maximum rate and is not sensitive to changes in traffic rate. This scenario, which we call MAX, is representative of the current commercial switches and is considered a baseline. The second configuration specifies a scenario that modulates bandwidth between $5 \mathrm{Mbps}, 10 \mathrm{Mbps}$ and the maximum rate. We set the model to report a power consumption of a port at $10 \mathrm{Mbps}$ to be $0.13 \mathrm{~W}$ and a port at $5 \mathrm{Mbps}$ to be $0.10 \mathrm{~W}$. These values are motivated by our measurements but reflect hypothetical hardware configurations. Power consumption is set to fixed values based on selected bandwidth.

To make bandwidth selection decisions, we configure the monitor to track the number of bytes transmitted and received on the active ports and then enforce modulation of the link rate with traffic shaping. The scenarios also specify a threshold over the transmit rates measured in the previous second of runtime to determine which link rate is selected for the next second. The threshold indicating that we need to move between $5 \mathrm{Mbps}$ and $10 \mathrm{Mbps}$ is set at $3 \mathrm{Mbps}$ and the threshold between $10 \mathrm{Mbps}$ and the maximum rate is $8 \mathrm{Mbps}$. We assume for this test that the power cost of modulating the rate is zero, the change between rates is instantaneous, and that our links require symmetric rates.

The third configuration highlights the capability of our extensions to support fine-grained dynamic power proportional experiments. In this configuration, we specify a scenario in which power consumption is directly proportional to the bytes that are transmitted. In this scenario, we specify a zero fixed cost for turning the device on and a $7.0 * 10^{-8} \mathrm{~J}$ cost per switched bit. This kind of device is not available today and may never be, but acts as an extreme case of the types of

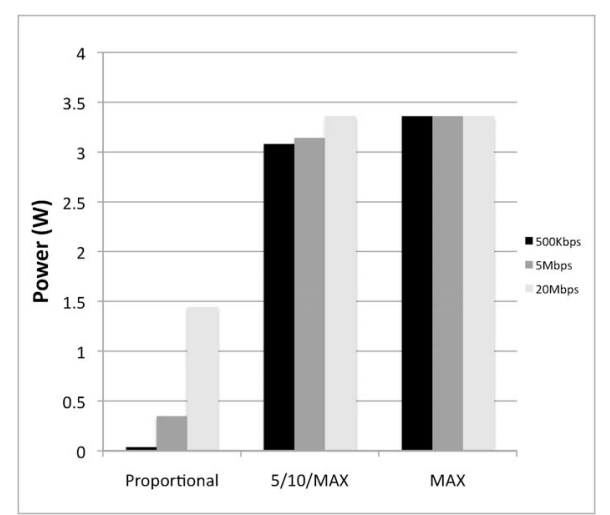

Fig. 2. Comparison of real time model power consumption between power proportional, 5/10/MAX, and MAX scenarios.

designs that can be investigated with our extensions. This scenario reads/net/proc/dev every $10 \mathrm{~ms}$ and sets the model accordingly. The power monitor reads the sensor values after sleeping for at least $100 \mathrm{~ms}$.

Each scenario is tested with three constant rate IPERF sources that generated traffic at $500 \mathrm{Kbps}, 5 \mathrm{Mbps}$, and $20 \mathrm{Mbps}$ respectively. We expect that given a constant rate of traffic, the testbed device will report lower power consumption for the scenarios with more rate/power options compared to the baseline MAX configuration.

In Figure 2 we report the modeled average power usage of the device during the bandwidth scaling tests. The results clearly show that the device with bandwidth scaling performs better than MAX and the device with bit-based power proportionality provides a dramatic improvement on power consumption. These results demonstrate that our extensions can indeed be used in a simple testbed to measure the relative power savings between different power-aware switch configurations and offer a unique new testbed capability. There are clearly many other possible configurations that are possible that would highlight the potential power savings if other components of the switch were power proportional.

Finally, we demonstrate the combined capabilities of our model/monitor and traffic shaper in a dynamic traffic scaling test. We configure a scenario in which we fix the maximum rate of the switch to be $5 \mathrm{Mbps}$, specify the cost per switched bit, measure / net/proc/dev every $10 \mathrm{~ms}$ and read the sensor values every $100 \mathrm{~ms}$. We then use the IPERF bandwidth estimation capability to scale up traffic from zero to what is available. Figure 3 shows the results of the test. We see a quick ramp up in power consumption proportional to the increase in IPERF traffic followed by a leveling of consumption at the value associated with the $5 \mathrm{Mbps}$ rate.

\section{CONCLUSIONS}

In this paper, we describe a set of extensions for Linuxbased switches that enable power-awareness experiments. The motivation for our work is the fact that there are currently very few options for experiments with new power-aware protocols and systems. Our extensions provide two key capabilities, 


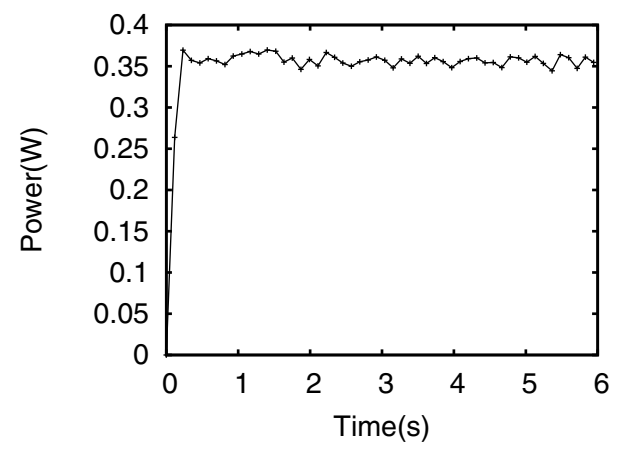

Fig. 3. Power consumption of power proportional model undergoing an IPERF bandwidth test with a maximum bandwidth set at $5 \mathrm{Mbps}$.

(i) the ability to model and monitor new power-aware techniques that are either fixed cost or proportional to system behavior, and (ii) a flexible traffic shaper that enables real time modulation of the switch to explore the design space of power-aware switching. We implement our extensions in an efficient software package that can be built and deployed on any Linux-enabled switch. Our implementation includes the ability to specify the details of behavior of the poweraware platform under test. We deployed our implementation on a simple switch to validate and demonstrate their capabilities. Our experiments highlight the fixed and variable cost features of our model along with its associated monitor that enables fine-grained measurements to be taken from the system. Our experiments also highlight the capabilities of our traffic shaper, which is based on the hierarchical token bucket in the TC utility in Linux. While the absolute values of the power measurements from our experiments are not necessarily important, they do show the how the relative impact of power proportional methods can be assessed. Our current work is focused on developing a set of standard power models and scenarios that can be used by researchers for a broad range of power-awareness experiments.

\section{ACKNOWLEDGEMENTS}

This work was supported in part by NSF grants CNS0831427 and CNS-0905186. Any opinions, findings, conclusions or other recommendations expressed in this material are those of the authors and do not necessarily reflect the view of the NSF.

\section{REFERENCES}

[1] The linksys wrt54gl wireless router. http://www.linksysbycisco.com, 2010.

[2] Net-SNMP. http://www.net-snmp.org, 2010.

[3] OpenWRT. http://openwrt.org, 2010.

[4] The Emulab Testbed. http://www.emulab.net, 2010.

[5] The Network Simulator. http://www.isi.edu/nsnam/ns, 2010.

[6] M. Allman, K. Christensen, B. Nordman, and V. Paxson. Enabling an Energy-efficient Future Internet Through Selectively Connected End Systems. In Proceedings of USENIX HotNets '07, Atlanta, GA, November 2007.

[7] A. Bavier, M. Bowman, B. Chun, D. Culler, S. Karlin, S. Muir, L. Peterson, T. Roscoe, T. Spalink, and M. Wawrzoniak. Operating System Support for Planetary-Scale Network Services. In Proceedings of USENIX NSDI '04, San Francisco, CA, March 2004.
[8] J. Broch, D. Maltz, D. Johnson, Y. Hu, and J. Jetcheva. A Performance Comparison of Multi-hop Wireless Ad Hoc Network Routing Protocols. In Proceedings of ACM MOBICOM '98, Dallas, TX, October 1998.

[9] J. Chabarek, J. Sommers, P. Barford, C. Estan, D. Tsiang, and S. Wright. Power Awareness in Network Design and Routing. In Proceedings of IEEE INFOCOM '08, Phoenix, AZ, April 2008.

[10] M. Gupta and S. Singh. Greening of the Internet. In Proceedings of ACM SIGCOMM '03, Karlsruhe, Germany, August 2003.

[11] B. Heller, S. Seetharaman, P. Mahadevan, Y. Yiakoumis, P. Sharma, S. Banerjee, and N. McKeown. ElasticTree: Saving Energy in Data Center Networks. In Proceedings of USENIX Symposium on Network System Design and Implementation (NSDI '10, San Jose, CA, April 2010.

[12] P. Mahadevan, P. Sharma, S. Banerjee, and P. Ranganathan. Energy Aware Network Operations. In IFIP Networking, May 2009.

[13] N. McKeown, T. Anderson, G. Parulkar, L. Peterson, J. Rexford, S. Shenker, and J. Turner. Openflow: Enabling innovations in campus networks. http://www.openflowswitch.org//documents/openflow-wplatest.pdf, 2010.

[14] S. Nedevschi, L. Popa, G. Iannaccone, S. Ratnasamy, and D. Wetherall. Reducing Network Energy Consumption via Sleeping and Rate Adaptation. In Proceedings of USENIX NSDI '08, San Francisco, CA, April 2008.

[15] C. Systems. Energywise technology white paper. http://www.cisco.com/en/US/products/ps10195, 2010. 